\title{
First-Order Orbit Queries
}

\author{
Shaull Almagor · Joël Ouaknine • James \\ Worrell
}

Received: date / Accepted: date

\begin{abstract}
Orbit Problems are a class of fundamental reachability questions that arise in the analysis of discrete-time linear dynamical systems such as automata, Markov chains, recurrence sequences, and linear while loops. Instances of the problem comprise a dimension $d \in \mathbb{N}$, a square matrix $A \in \mathbb{Q}^{d \times d}$, and a query regarding the behaviour of some sets under repeated applications of $A$. For instance, in the Semialgebraic Orbit Problem, we are given semialgebraic source and target sets $S, T \subseteq \mathbb{R}^{d}$, and the query is whether there exists $n \in \mathbb{N}$ and $x \in S$ such that $A^{n} x \in T$.

The main contribution of this paper is to introduce a unifying formalism for a vast class of orbit problems, and show that this formalism is decidable for dimension $d \leq 3$. Intuitively, our formalism allows one to reason about any firstorder query whose atomic propositions are a membership queries of orbit elements in semialgebraic sets. Our decision procedure relies on separation bounds for algebraic numbers as well as a classical result of transcendental number theory-Baker's theorem on linear forms in logarithms of algebraic numbers. We moreover argue

Joël Ouaknine is supported by ERC grant AVS-ISS (648701) and by DFG grant 389792660 as part of TRR 248 (see https://perspicuous-computing.science). James Worrell is supported by EPSRC Fellowship EP/N008197/1. Shaull Almagor has received funding from the European Union's Horizon 2020 research and innovation programme under the Marie Skłodowska-Curie grant agreement No 837327.
\end{abstract}

Shaull Almagor

Computer Science Department

Technion, Israel

E-mail: shaull@cs.technion.ac.il

Joël Ouaknine

Max Planck Institute for Software Systems,

Germany,

and Department of Computer Science

Oxford University, UK

E-mail: joel@mpi-sws.org

James Worrell

Department of Computer Science

Oxford University, UK

E-mail: jbw@cs.ox.ac.uk 
that our main result represents a natural limit to what can be decided (with respect to reachability) about the orbit of a single matrix. On the one hand, semialgebraic sets are arguably the largest general class of subsets of $\mathbb{R}^{d}$ for which membership is decidable. On the other hand, previous work has shown that in dimension $d=4$, giving a decision procedure for the special case of the Orbit Problem with singleton source set $S$ and polytope target set $T$ would entail major breakthroughs in Diophantine approximation. 


\section{Introduction}

Given $d \in \mathbb{N}$, a square matrix $A \in \mathbb{Q}^{d \times d}$, and a point $s \in \mathbb{R}^{d}$, the orbit of $s$ under $A$ is the infinite sequence $\left\langle s, A s, A^{2} s, A^{3} s, \ldots\right\rangle$. Given a target set $T$, a classical decision problem is whether there exists $n \in \mathbb{N}$ such that $A^{n} s \in T$. One way to categorise such problems is according to the types of sets allowed for the source and target (e.g., polytopes or semialgebraic sets). We collectively refer to the various problems that arise in this way as Orbit Problems. Orbit Problems occur naturally in the reachability analysis of discrete-time linear dynamical systems, including Markov chains, automata, recurrence sequences, and linear loops in program analysis (see $[6$, 13,10] and references therein).

In order to describe the main result of this paper in relation to existing work, we identify three successively more general types of Orbit Problems. In the pointto-point version both the source and target are singletons with rational coordinates; in the Polytopic Orbit Problem the source and target $S$ and $T$ are polytopes (i.e., sets defined by conjunctions of linear inequalities with rational coefficients); in the Semialgebraic Orbit Problem $S$ and $T$ are semialgebraic sets defined with rational parameters.

The question of the decidability of the Point-to-Point Orbit Problem was raised by Harrison in 1969 [11]. The problem remained open for ten years until it was finally resolved in a seminal paper of Kannan and Lipton [13], who in fact gave a polynomial-time decision procedure.

The Polytopic Orbit Problem is considerably more challenging than the pointto-point version, and its decidablity seems out of reach for now. Indeed the special case in which $S$ is a singleton and $T$ is a linear subspace of $\mathbb{R}^{d}$ of dimension $d-1$ is a well-known decision problem in its own right, called the Skolem Problem, whose decidability has been open for many decades [22]. In contrast to the point-to-point case the only positive decidability results for the Polytopic Orbit Problem are in the case of fixed dimension $d$. For the Skolem Problem, decidability is known for $d \leq 4[16,24]$. In case $S$ and $T$ are allowed to be arbitrary polytopes, decidability is known in case $d \leq 3$ [1] (see also [5]). While Kannan and Lipton's decision procedure in the point-to-point case mainly relied on algebraic number theory (e.g., separation bounds between algebraic numbers and prime factorisation of ideals in rings of algebraic integers), the decision procedures for the Skolem Problem and the Polytopic Orbit Problem additionally use results from transcendental number theory (specifically Baker's theorem about linear forms in logarithms of algebraic numbers). It was shown in [5] that the existence of a decision procedure for the Polytopic Orbit Problem in dimension $d=4$ would entail computability of the Diophantine approximation types of a general class of transcendental numbers (a problem considered intractable at present). Not only does this suggest that the use of sophisticated transcendental tools is unavoidable in analysing the Polytopic Orbit Problem, it also indicates that further progress beyond the case $d=3$ is contingent upon significant advances in the field of Diophantine approximation.

In a preliminary version of this paper [2], we remained in dimension $d=3$ and considered a generalisation of previous work by allowing the source and target sets to be semialgebraic, that is, defined by Boolean combinations of polynomial equalities and inequalities. This allowed us to handle three-dimensional source and target sets in much greater geometrical generality than polytopes. In applications to program analysis and dynamical systems, semialgebraic sets are indispensable 
to formulate sufficiently expressive models (e.g., to describe initial conditions and transition guards) and in model analysis (e.g., in synthesising invariants and barrier certificates and approximating sets of reachable states) $[17,14]$. As we elaborate below, in this paper we present a unifying approach to the problems above, which enables us to handle much richer settings.

Revisiting the above problems, we can formulate the types of orbit queries as follows: the Point-to-Point Orbit Problem is given by the sentence $\exists n \in \mathbb{N}$. $A^{n} s=t$, and the Polytopic and Semialgebraic Orbit Problems can be written as $\exists n \in \mathbb{N} \exists x \in \mathbb{R}^{d} . x \in S \wedge A^{n} x \in T$. In both cases, we essentially ask whether there exists a stage $n$ of the orbit for which some first-order condition holds, where the condition is allowed to specify membership queries in, e.g., semialgebraic sets.

In this work, we generalize the above view (and in particular our results in [2]) and provide a specification formalism for first-order orbit queries, which subsumes the Semialgebraic Orbit Problem, as well as other interesting queries, and unifies all the above problems. Our main technical result is establishing the decidability of our formalism for dimension $d=3$. Formally, our queries have the form $\exists n \in \mathbb{N} . \Phi(n)$, where $\Phi(n)$ is a first-order sentence that may include atomic propositions of the form $A^{n} x \in T$, for semialgebraic $T$.

As in previous work on the Skolem Problem and on the Polytopic Orbit Problem, Baker's Theorem plays a crucial role in the present development. Technically, there are two main difficulties in generalising from the polytopic case to general queries involving first-order (i.e., semialgebraic) statements. The first difficulty lies in the delicate analytic arguments that are required to bring Baker's Theorem to bear. More precisely: (i) we need to resort to symbolic quantifier elimination (in lieu of explicit Fourier-Motzkin elimination, which had been used in the Polytopic Orbit Problem), since we are now dealing with non-linear constraints; (ii) we also need to perform spectral calculations symbolically, via the use of Vandermonde methods, instead of the explicit direct approach possible in our earlier work; and (iii) we replace triangulation of polytopes by cylindrical algebraic decomposition of semialgebraic sets into cells, which again necessitates a new symbolic treatment along with a substantially refined analysis based on Taylor approximation of the attendant functions.

In summary, this paper provides a decision procedure for a vast family of Orbit Problems in dimension $d=3$, involving semialgebraic sets. The latter appears to be a natural limit to the positive decidability results that can be obtained for such problems, barring major new advances in Diophantine approximation.

On a technical level, our contributions are threefold: in Section 3 we introduce our model and demonstrate its expressive power. We then formulate the decision problem at hand, and state our main theorem. In Section 4 we start by analysing a quantifier-free version of the problem, namely the case where only queries of the form $A^{n} s \in T$ for fixed $s$ and $T$ are allowed. We then reduce this problem in Section 4.1 to solving certain systems of polynomial-exponential equalities and inequalities, and in Section 4.2 we show precisely how to solve such systems. The second technical contribution consists in handling the general case of the problem, in Section 5. There, we show how to circumvent problems that arise in the presence of quantifiers, and arrive at a system that can ultimately be solved using the techniques and results developed in Section 4.2. 


\section{Mathematical Tools}

In this section we introduce the key technical tools used in this paper.

\subsection{Algebraic numbers}

For $p \in \mathbb{Z}[x]$ a polynomial with integer coefficients, we denote by $\|p\|$ the bit length of its representation as a list of coefficients encoded in binary. Note that the degree of $p$, denoted $\operatorname{deg}(p)$ is at most $\|p\|$, and the height of $p$ - i.e., the maximum of the absolute values of its coefficients, denoted $H(p)$ - is at most $2^{\|p\|}$.

We begin by summarising some basic facts about the field of algebraic numbers (denoted A) and (efficient) arithmetic therein. The main references include $[4,7$, 21]. A complex number $\alpha$ is algebraic if it is a root of a single-variable polynomial with integer coefficients. The defining polynomial of $\alpha$, denoted $p_{\alpha}$, is the unique polynomial of least degree, and whose coefficients do not have common factors, which vanishes at $\alpha$. The degree and height of $\alpha$ are respectively those of $p_{\alpha}$, and are denoted $\operatorname{deg}(\alpha)$ and $H(\alpha)$. A standard representation ${ }^{1}$ for algebraic numbers is to encode $\alpha$ as a tuple comprising its defining polynomial together with rational approximations of its real and imaginary parts of sufficient precision to distinguish $\alpha$ from the other roots of $p_{\alpha}$. More precisely, $\alpha$ can be represented by $\left(p_{\alpha}, a, b, r\right) \in \mathbb{Z}[x] \times \mathbb{Q}^{3}$ provided that $\alpha$ is the unique root of $p_{\alpha}$ inside the circle in $\mathbb{C}$ of radius $r$ centred at $a+b i$. A separation bound due to Mignotte [15] asserts that for roots $\alpha \neq \beta$ of a polynomial $p \in \mathbb{Z}[x]$, we have

$$
|\alpha-\beta|>\frac{\sqrt{6}}{d^{(d+1) / 2} H^{d-1}}
$$

where $d=\operatorname{deg}(p)$ and $H=H(p)$. Thus if $r$ is required to be less than a quarter of the root-separation bound, the representation is well-defined and allows for equality checking. Given a polynomial $p \in \mathbb{Z}[x]$, it is well-known how to compute standard representations of each of its roots in time polynomial in $\|p\|[4,7,19]$. Thus given an algebraic number $\alpha$ for which we have (or wish to compute) a standard representation, we write $\|\alpha\|$ to denote the bit length of this representation. From now on, when referring to computations on algebraic numbers, we always implicitly refer to their standard representations.

Note that Equation (1) can be used more generally to separate arbitrary algebraic numbers: indeed, two algebraic numbers $\alpha$ and $\beta$ are always roots of the polynomial $p_{\alpha} p_{\beta}$ of degree at $\operatorname{most} \operatorname{deg}(\alpha)+\operatorname{deg}(\beta)$, and of height at most $H(\alpha) H(\beta)$. Given algebraic numbers $\alpha$ and $\beta$, one can compute $\alpha+\beta, \alpha \beta, 1 / \alpha$ (for $\alpha \neq 0$ ), $\bar{\alpha}$, and $|\alpha|$, all of which are algebraic, in time polynomial in $\|\alpha\|+\|\beta\|$. Likewise, it is straightforward to check whether $\alpha=\beta$. Moreover, if $\alpha \in \mathbb{R}$, deciding whether $\alpha>0$ can be done in time polynomial in $\|\alpha\|$. Efficient algorithms for all these tasks can be found in $[4,7]$.

\footnotetext{
1 Note that this representation is not unique.
} 
2.2 First-order theory of the reals

Let $\vec{x}=x_{1}, \ldots, x_{m}$ be a list of $m$ real-valued variables, and let $\sigma(\vec{x})$ be a Boolean combination of atomic predicates of the form $g(\vec{x}) \sim 0$, where each $g(\vec{x}) \in \mathbb{Z}[\vec{x}]$ is a polynomial with integer coefficients over these variables, and $\sim \in\{>,=\}$. A formula of the first-order theory of the reals is of the form $Q_{1} x_{1} Q_{2} x_{2} \cdots Q_{m} x_{m} \sigma(\vec{x})$, where each $Q_{i}$ is one of the quantifiers $\exists$ or $\forall$. Let us denote the above formula by $\tau$, and write $\|\tau\|$ to denote the bit length of its syntactic representation. Tarski famously showed that the first-order theory of the reals is decidable [23]. His procedure, however, has non-elementary complexity. Many substantial improvements followed over the years, starting with Collins's technique of cylindrical algebraic decomposition [8], and culminating with the fine-grained analysis of Renegar [21]. In this paper, we will use the following theorems $[20,21]$.

Theorem 1 (Renegar [20]) The problem of deciding whether a closed formula $\tau$ of the form above holds over the reals is in $2 \boldsymbol{E X} \boldsymbol{P}$, and in PSPACE if $\tau$ has only existential quantifiers.

Theorem 2 (Renegar [21]) There is an algorithm that, given a formula $\tau\left(x_{1}, \ldots, x_{m}\right)$ where $x_{1}, \ldots, x_{m}$ are free variables, computes an equivalent quantifier-free formula in disjunctive normal form $(D N F) \Phi\left(x_{1}, \ldots, x_{m}\right)=\bigvee_{I} \wedge_{J} R_{I, J}\left(x_{1}, \ldots, x_{m}\right) \sim_{I, J} 0$ where $R_{I, J}$ is a polynomial ${ }^{2}$ and $\sim_{I, J} \in\{>,=\}$. Moreover, the algorithm runs in time $2^{2^{\mathcal{O}(\|\tau\|)}}$, and in particular, $\|\Phi\|=2^{2^{\mathcal{O}(\|\tau\|)}}$.

A set $S \subseteq \mathbb{R}^{d}$ is semialgebraic if there exists a formula $\Phi\left(x_{1}, \ldots, x_{d}\right)$ in the firstorder theory of the reals with free variables $x_{1}, \ldots, x_{d}$ such that $S=\left\{\left(c_{1}, \ldots, c_{d}\right)\right.$ : $\Phi\left(c_{1}, \ldots, c_{d}\right)$ is true $\}$.

We remark that algebraic constants can also be incorporated as coefficients in the first-order theory of the reals (and in particular, in the definition of semialgebraic sets), as follows. Consider a polynomial $g\left(x_{1}, \ldots, x_{m}\right)$ with algebraic coefficients $c_{1}, \ldots, c_{k}$. We replace every $c_{j}$ with a new, existentially-quantified variable $y_{j}$, and add to the sentence the predicates $p_{c_{j}}\left(y_{j}\right)=0$ and $\left(y_{j}-(a+b i)\right)^{2}<r^{2}$, where $\left(p_{c_{j}}, a, b, r\right)$ is the representation of $c_{j}$. Then, in any evaluation of this formula to True, it must hold that $y_{j}$ is assigned value $c_{j}$.

\section{First-Order Orbit Queries}

In this section we introduce and motivate the model of First-Order Orbit Queries. Given a matrix $A \in \mathbb{Q}^{d \times d}$, a first-order orbit query (FOOQ) over $A$ is a sentence of the form $\exists n \in \mathbb{N} . \Phi(n)$, where $\Phi(n)$ is a fully quantified first-order sentence whose atomic propositions are either of the form $p(\vec{x}) \geq 0$ for a polynomial $p \in \mathbb{Z}[\vec{x}]$, or of the form $A^{n} \vec{x} \in T$ for a semialgebraic set $T$. We refer to the latter form as orbit propositions. Note that the matrix $A$ is fixed for the query, but there can be multiple semialgebraic sets at play. In the following we omit $A$ when it is clear from context.

Clearly, FOoQs subsume the first-order theory of the reals. In particular, we can express queries such as $\vec{x} \in S$ for a semialgebraic set $S$.

We illustrate this formalism with some important examples.

\footnotetext{
${ }^{2}$ Technically, the indices should be $I, J_{I}$, but we omit the dependency of $J$ on $I$ for simplicity.
} 
Example 1 (The Kannan-Lipton Orbit Problem) The Kannan-Lipton Orbit Problem $[12,13]$ described in Section 1 can be formulated as the FoOQ $\exists n \in \mathbb{N} . A^{n} s \in\{t\}$, where $s, t \in \mathbb{Q}^{d}$ are the initial and target vectors, respectively. Observe that this is a "quantifier-free" FOOQ, since both $s$ and $t$ are fixed.

Example 2 (The Semialgebraic Collision Problem) The Semialgebraic Collision Problem, described in Section 1, can also be formulated as a FOOQ: given semialgebraic sets $S, T \subseteq \mathbb{R}^{d}$, we have the FOOQ $\exists n \in \mathbb{N} \exists x \in \mathbb{Q}^{d} . x \in S \wedge A^{n} x \in T$.

Example 2 is the original problem studied in a preliminary version of this work [2]. However, FOOQs are much more general, as we now illustrate.

Example 3 (The Semialgebraic Parking Problem) A dual version of the Semialgebraic Collision Problem asks, given semialgebraic sets $S, T$ and a matrix $A$, whether $A^{n} S \subseteq T$ for some $n \in \mathbb{N}$. That is, whether the entire set $S$ becomes contained in $T$ after some applications of $A$ (intuitively, does $S$ "park" within $T$ during its orbit). We can formulate this as follows: $\exists n \in \mathbb{N} \forall x \in \mathbb{Q}^{d} . x \in S \rightarrow A^{n} x \in T$.

Example 4 (Near-Coverage Parking) A less-restrictive version of the Semialgebraic Parking Problem asks the following: given semialgebraic sets $S, T$, a matrix $A$, and $\epsilon \geq 0$, does there exist $n \in \mathbb{N}$ such that for every point $x \in S$, there is another point $y \in \mathbb{R}^{d}$ that is at distance at most $\epsilon$ from $x$, and for which $A^{n} y \in T$ (for $\epsilon=0$ this is exactly the Parking Problem). This is formulated as follows: $\exists n \in \mathbb{N} \forall x \in \mathbb{Q}^{d} \exists y \in \mathbb{Q}^{d} .|y-x| \leq \epsilon \wedge\left(x \in S \rightarrow A^{n} y \in T\right)$.

The fundamental decision problem for FooQs is determining the truth valuegiven a FOOQ $\exists n \in \mathbb{N} . \Phi(n)$, decide whether it holds. We dub this simply the FOOQ Problem.

Note that when the FOOQ Problem is decidable, we can trivially also decide whether the negation of a FOOQ is true, namely whether $\forall n \in \mathbb{N} . \neg \Phi(n)$ holds. This enables us to handle universal quantification on $n$, as well as existential.

As explained in Section 1, for dimension $d>3$, the decidability status of even simple fragments of the FOOQ Problem are open. Thus, we henceforth restrict attention to the case $d=3$.

For uniformity, we assume without loss of generality that the quantifiers in $\Phi(n)$ are pushed outside. Thus, $\Phi(n)$ is of the form $Q_{1} x_{1}, \ldots, Q_{m} x_{m} . \Psi\left(n, x_{1}, \ldots, x_{m}\right)$, where each $Q_{i}$ is either $\exists$ or $\forall$, the $x_{i}$ are real-valued variables, and $\Psi$ is a quantifierfree formula whose atomic propositions are as above.

Our main result is the following.

Theorem 3 The FOOQ Problem is decidable.

We prove Theorem 3 in an incremental fashion: in Section 4 we handle a restricted version of the problem, where $\Phi(n)$ is quantifier-free. Then, in Section 5 we show how to eliminate quantifiers from $\Phi(n)$, thus reducing the general case to the quantifier-free case.

\section{Almost Self-Conjugate Systems of Inequalities}

In this section we lay the groundwork for solving the FOOQ Problem in dimension 3 . We do so by initially treating the case where the formula $\Phi(n)$ is quantifier free. 


\subsection{Analysis of the Quantifier-Free First-Order Orbit Query Problem}

In the quantifier-free FOOQ Problem, we are given a query of the form $\exists n \in \mathbb{N} \Phi(n)$ and a matrix $A$, where $\Phi(n)$ is a Boolean combination of expressions in the following forms:

1. $g(\vec{s}) \sim 0$ where $g \in \mathbb{Z}[\vec{x}], \vec{s} \in(\mathbb{A} \cap \mathbb{R})^{3}$ is a vector with real-algebraic ${ }^{3}$ entries $^{2}$ and $\sim \in\{>,=\}$.

2. $A^{n} \vec{s} \in T$ where $s \in(\mathbb{A} \cap \mathbb{R})^{3}$ and $T \subseteq \mathbb{R}^{3}$ is a semialgebraic set.

and we wish to determine whether the query holds.

First, note that expressions of the first form do not depend on $n$, and can thus be resolved and replaced with true or false. We thus assume that no such expressions exist in $\Phi(n)$.

Let $T \subseteq \mathbb{R}^{d}$ be a semialgebraic set such that $A^{n} \vec{s} \in T$ is an orbit proposition in $\Phi(n)$ (recall that there may be several such $\vec{s}$ and $T$ pairs). By Theorem 2, we can compute a quantifier-free representation of $T$. That is, we can write $T=\{(x, y, z)$ : $\left.\bigvee_{I} \bigwedge_{J} R_{I, J}(x, y, z) \sim_{I, J} 0\right\}$ where $R_{I, J}$ are polynomials with integer coefficients, and $\sim_{I, J} \in\{>,=\}$. Note that then, $A^{n} \vec{s} \in T$ iff $\bigvee_{I} \wedge_{J} R_{I, J}\left(A^{n} \vec{s}\right) \sim_{I, J} 0$

Applying this for each orbit proposition $A^{n} \vec{s} \in T$ in $\Phi(n)$, and transforming $\Phi(n)$ to disjunctive normal form, we can write (abusing the indices $I, J$ and the notation $\left.R_{I, J}\right) \Phi(n)=\bigvee_{I} \bigwedge_{J} R_{I, J}\left(A^{n} \vec{s}_{I, J}\right) \sim_{I, J} 0$, with $\sim_{I, J} \in\{>,=\}$.

For the purpose of solving the FOOQ Problem, we note that it is enough to consider each disjunct separately. Thus, we can assume $\Phi(n)=\bigwedge_{J} R_{J}\left(A^{n} \vec{s}_{J}\right) \sim_{J} 0$ and it remains to decide whether $\exists n \in \mathbb{N} \Phi(n)$.

Next, consider the eigenvalues of $A$. Since $A$ is a $3 \times 3$ matrix, then either it has only real eigenvalues, or it has one real eigenvalue and two conjugate complex eigenvalues. In particular, if $A$ has complex eigenvalues, then it is diagonalisable.

The case where $A$ has only real eigenvalues is treated in Appendix A, and is considerably simpler.

Henceforth, we assume $A$ has complex eigenvalues, so that $A=P D P^{-1}$ with $D=\left(\begin{array}{ccc}\lambda & 0 & 0 \\ 0 & \bar{\lambda} & 0 \\ 0 & 0 & \rho\end{array}\right)$, where $\lambda$ is a complex eigenvalue, $\rho \in \mathbb{R}$, and $P$ an invertible matrix.

Observe that $A^{n}=P D^{n} P^{-1}$. By carefully analysing the structure of $P$, it is not hard to show that for every $\vec{s} \in(\mathbb{A} \cap \mathbb{R})^{3}$, we have that $A^{n} s=\left(\begin{array}{l}a_{1} \lambda^{n}+\overline{a_{1}} \bar{\lambda}^{n}+b_{1} \rho^{n} \\ a_{2} \lambda^{n}+\overline{a_{2}} \bar{\lambda}^{n}+b_{2} \rho^{n} \\ a_{3} \lambda^{n}+\overline{a_{3}} \bar{\lambda}^{n}+b_{3} \rho^{n}\end{array}\right)$ where the $a_{i}$ and $b_{i}$ are algebraic (and depend on $\vec{s}$ and $A$ ) and the $b_{i}$ are also real (see Appendix $\mathrm{C}$ for a detailed analysis).

Thus, we want to decide whether there exists $n \in \mathbb{N}$ such that $R_{J}\left(a_{1} \lambda^{n}+\right.$ $\left.\overline{a_{1}} \bar{\lambda}^{n}+b_{1} \rho^{n}, a_{2} \lambda^{n}+\overline{a_{2}} \bar{\lambda}^{n}+b_{2} \rho^{n}, a_{3} \lambda^{n}+\overline{a_{3}} \bar{\lambda}^{n}+b_{3} \rho^{n}\right) \sim_{J} 0$ for every $J$ (Observe that $a_{1}, a_{2}, a_{3}, b_{1}, b_{2}, b_{3}$ may also depend on $J$, but we omit that for readability).

Since $R_{J}$ is a polynomial, then by aggregating coefficients we can write, for every $\vec{s} \in(\mathbb{A} \cap \mathbb{R})^{d}$,

$$
R_{J}\left(A^{n} \vec{s}\right)=\sum_{0 \leq p_{1}, p_{2}, p_{3} \leq k} \alpha_{p_{1}, p_{2}, p_{3}} \lambda^{n p_{1}} \bar{\lambda}^{n p_{2}} \rho^{n p_{3}}+\overline{\alpha_{p_{1}, p_{2}, p_{3}}} \bar{\lambda}^{n p_{1}} \lambda^{n p_{2}} \rho^{n p_{3}}
$$

3 Formally, only rational numbers should be allowed. However, the results of this section are meant to apply for a formula obtained by removing quantifiers in a general FOOQ. Thus, we must account for algebraic numbers as well. 
for some $k \in \mathbb{N}$, where the $\alpha$ 's depend on $\vec{s}, A$ and $R_{J}$. Note that we treat the (real) coefficients of $\rho$ as a sum of complex conjugate coefficients, but this can easily be achieved by writing e.g., $c \rho^{n p}=\frac{c}{2} \rho^{n p}+\frac{c}{2} \rho^{n p}$.

We notice that the polynomial $R_{J}\left(A^{n} \vec{s}\right)$, consists of conjugate summands. More precisely, $R\left(A^{n} \vec{s}\right)$, when viewed as a polynomial in $\lambda^{n}, \bar{\lambda}^{n}$, and $\rho^{n}$, has the following property.

Property 1 (Almost Self-Conjugate Polynomial) A complex polynomial $Q\left(z_{1}, z_{2}, z_{3}\right)$ over $\mathbb{C}^{3}$ is almost self-conjugate if

$$
Q\left(z_{1}, z_{2}, z_{3}\right)=\sum_{0 \leq t_{1}, t_{2}, t_{3} \leq \ell} \delta_{t_{1}, t_{2}, t_{3}} z_{1}^{t_{1}} z_{2}^{t_{2}} z_{3}^{t_{3}}+\overline{\delta_{t_{1}, t_{2}, t_{3}}} z_{2}^{t_{1}} z_{1}^{t_{2}} z_{3}^{t_{3}} .
$$

That is, if $z_{2}=\overline{z_{1}}$ and $z_{3}$ is a real variable, then the monomials in $Q$ appear in conjugate pairs with conjugate coefficients.

We refer to the conjunction $\bigwedge_{J} R_{J}\left(A^{n} \vec{s}\right) \sim_{J} 0$ as an almost self-conjugate system. It remains to show that we can decide whether there exists $n \in \mathbb{N}$ that solves the system.

\subsection{Solving Almost Self-Conjugate Systems}

Our starting point is now an almost self-conjugate system as described above. In the following, we will consider a single conjunct $R_{J}\left(A^{n} \vec{s}\right) \sim_{J} 0$.

We start by normalising the expression $R_{J}\left(A^{n} \vec{s}\right) \sim_{J} 0$ in the form of (2), as follows. Let $\Lambda=\max \left\{\left|\lambda^{p_{1}} \bar{\lambda}^{p_{2}} \rho^{p_{3}}\right|: \alpha_{p_{1}, p_{2}, p_{3}} \neq \emptyset\right\}$, we divide the expression in (2) by $\Lambda^{n}$, and get that $R_{J}\left(A^{n} \vec{s}\right) \sim_{J} 0$ iff

$$
\sum_{m=0}^{k} \beta_{m} \gamma^{n m}+\overline{\beta_{m}} \bar{\gamma}^{n m}+r(n) \sim_{J} 0
$$

where the $\beta_{m}$ are algebraic coefficients, $\gamma=\frac{\lambda}{|\lambda|}$ satisfies $|\gamma|=1$ and $r(n)=$ $\sum_{l=1}^{k^{\prime}} \chi_{l} \mu_{l}^{n}+\bar{\chi}_{l} \mu_{l}^{n}$ with $\chi_{l}$ being algebraic coefficients, and $\left|\mu_{l}\right|<1$ for every $1 \leq l \leq k^{\prime}$. Moreover, every $\mu_{l}$ is a quotient of two elements of the form $\lambda^{p_{1}} \bar{\lambda}^{p_{2}} \rho^{p_{3}}$, and thus, by Section 2.1, $\operatorname{deg}\left(\mu_{l}\right)=\left\|R_{J}\right\|^{\mathcal{O}(1)}$ and $H\left(\mu_{l}\right)=2^{\left\|R_{J}\right\|^{\mathcal{O}(1)}}$. Note that for simplicity, we reuse the number $k$, although it may differ from $k$ in (2). We refer to Equation (3) as the normalised expression.

In the following, we assume that at least one of the $\beta_{j}$ is nonzero for $j \geq 1$. Indeed, otherwise we can recast our analysis on $r(n)$, which is of lower order.

We now split our analysis according to whether or not $\gamma$ is a root of unity. That is, whether $\gamma^{d}=1$ for some $d \in \mathbb{N}$.

\subsubsection{The case where $\gamma$ is a root of unity}

Suppose that $\gamma$ is a root of unity. Then, the set $\left\{\gamma^{n}: n \in \mathbb{N}\right\}$ is a finite set $\left\{\gamma^{0}, \ldots, \gamma^{d-1}\right\}$. Thus, by splitting the analysis of $A^{n} \vec{s}$ according to $n \bmod d$, we can reduce the problem to $d$ instances which involve only real numbers. In Appendix B we detail how to handle this case, and comment on its complexity. 
4.2.2 The case where $\gamma$ is not a root of unity

When $\gamma$ is not a root of unity, the set $\left\{\gamma^{n}: n \in \mathbb{N}\right\}$ is dense in the unit circle. With this motivation in mind, we define, for a normalised expression, its dominant function $f: \mathbb{C} \rightarrow \mathbb{R}$ as $f(z)=\sum_{m=0}^{k} \beta_{m} z^{m}+\bar{\beta}_{m} \bar{z}^{m}$. Observe that (3) is now equivalent to $f\left(\gamma^{n}\right)+r(n) \sim_{J} 0$.

Our main technical tool in handling (3) is the following lemma.

Lemma 1 Consider a normalised expression as in (3). Let $\|\mathcal{I}\|$ be its encoding length, and let $f$ be its dominant function. Then there exists $N \in \mathbb{N}$ computable in polynomial time in $\|\mathcal{I}\|$ with $N=2^{\|\mathcal{I}\|^{\mathcal{O}(1)}}$ such that for every $n>N$ it holds that

1. $f\left(\gamma^{n}\right) \neq 0$,

2. $f\left(\gamma^{n}\right)>0$ iff $f\left(\gamma^{n}\right)+r(n)>0$,

3. $f\left(\gamma^{n}\right)<0$ iff $f\left(\gamma^{n}\right)+r(n)<0$.

In particular, the lemma implies that if $f\left(\gamma^{n}\right)+r(n)=0$, then $n \leq N$. That is, if $\sim_{J}$ is "=", then there is a bound on $n$ that solves the system.

Remark 1 In the formulation of Lemma 1, we measure the complexity with respect to $\|\mathcal{I}\|$. However, recall that when the input is $T$, we actually have $\|\mathcal{I}\|=2^{2^{\mathcal{O}(\|T\|)}}$. The analysis in Lemma 1 thus allows us to separate the blowup required for analysing the semialgebraic target from our algorithmic contribution. In particular, when the target has bounded description length, we can obtain better complexity bounds.

We prove Lemma 1 in the remainder of this section.

Since $\left\{\gamma^{n}: n \in \mathbb{N}\right\}$ is dense on the unit circle, our interest in $f$ is also about the unit circle. By identifying $\mathbb{C}$ with $\mathbb{R}^{2}$, we can think of $f$ as a function of two real variables. In this view, $f(x, y)$ is a polynomial with algebraic coefficients, and we can therefore compute a description of the algebraic set $Z_{f}=$ $\left\{(x, y): f(x, y)=0 \wedge x^{2}+y^{2}=1\right\}$. We start by showing that this set is finite. Define $g:(-\pi, \pi] \rightarrow \mathbb{R}$ by $g(x)=f\left(e^{i x}\right)$. Explicitly, we have $g(x)=\sum_{m=0}^{k} 2\left|\beta_{m}\right| \cos (m x+$ $\left.\theta_{m}\right)$ where $\theta_{m}=\arg \left(\beta_{m}\right)$. Clearly there is a one-to-one correspondence between $Z_{f}$ and the roots of $g$.

We present the following proposition, which will be reused later in the proof.

Proposition 1 For every $x \in(-\pi, \pi]$ there exists $1 \leq j \leq 4 k$ such that $g^{(j)}(x) \neq 0$, where $g^{(j)}$ is the $j$-th derivative of $g$.

Proof Assume by way of contradiction that $g^{\prime}(x)=\ldots=g^{4 k}(x)=0$. For every $1 \leq j \leq 4 k$ we have that

$$
g^{(j)}(x)= \begin{cases}\sum_{m=1}^{k} m^{j} 2\left|\beta_{m}\right| \cos \left(m x+\theta_{m}\right) & j \equiv_{4} 0 \\ \sum_{m=1}^{k}-m^{j} 2\left|\beta_{m}\right| \sin \left(m x+\theta_{m}\right) & j \equiv_{4} 1 \\ \sum_{m=1}^{k}-m^{j} 2\left|\beta_{m}\right| \cos \left(m x+\theta_{m}\right) & j \equiv_{4} 2 \\ \sum_{m=1}^{k} m^{j} 2\left|\beta_{m}\right| \sin \left(m x+\theta_{m}\right) & j \equiv_{4} 3\end{cases}
$$

(note that the summand that corresponds to $m=0$ is dropped out in the derivative, as it is constant). 
Splitting according $j \bmod 4$, we rewrite the equations $g^{(j)}(x)=0$ in matrix form as follows. ${ }^{4}$

$$
\text { for } j \equiv_{4} 0: \quad\left(\begin{array}{cccc}
1^{4} & 2^{4} & \cdots & k^{4} \\
1^{8} & 2^{8} & \cdots & k^{8} \\
\vdots & \vdots & \vdots & \vdots \\
1^{4 k} & 2^{4 k} & \cdots & k^{4 k}
\end{array}\right)\left(\begin{array}{c}
2\left|\beta_{1}\right| \cos \left(x+\theta_{1}\right) \\
2\left|\beta_{2}\right| \cos \left(2 x+\theta_{2}\right) \\
\vdots \\
2\left|\beta_{k}\right| \cos \left(k x+\theta_{k}\right)
\end{array}\right)=\left(\begin{array}{c}
0 \\
0 \\
\vdots \\
0
\end{array}\right)
$$

$$
\begin{aligned}
& \text { for } j \equiv_{4} 1: \quad\left(\begin{array}{cccc}
-1^{1} & -2^{1} & \cdots & -k^{1} \\
-1^{5} & -2^{5} & \cdots & -k^{5} \\
\vdots & \vdots & \vdots & \vdots \\
-1^{4 k-3} & -2^{4 k-3} & \cdots & -k^{4 k-3}
\end{array}\right)\left(\begin{array}{c}
2\left|\beta_{1}\right| \sin \left(x+\theta_{1}\right) \\
2\left|\beta_{2}\right| \sin \left(2 x+\theta_{2}\right) \\
\vdots \\
2\left|\beta_{k}\right| \sin \left(k x+\theta_{k}\right)
\end{array}\right)=\left(\begin{array}{c}
0 \\
0 \\
\vdots \\
0
\end{array}\right) \\
& \text { for } j \equiv_{4} 2: \quad\left(\begin{array}{cccc}
-1^{2} & -2^{2} & \cdots & -k^{2} \\
-1^{6} & -2^{6} & \cdots & -k^{6} \\
\vdots & \vdots & \vdots & \vdots \\
-1^{4 k-2} & -2^{4 k-2} & \cdots & -k^{4 k-2}
\end{array}\right)\left(\begin{array}{c}
2\left|\beta_{1}\right| \cos \left(x+\theta_{1}\right) \\
2\left|\beta_{2}\right| \cos \left(2 x+\theta_{2}\right) \\
\vdots \\
2\left|\beta_{k}\right| \cos \left(k x+\theta_{k}\right)
\end{array}\right)=\left(\begin{array}{c}
0 \\
0 \\
\vdots \\
0
\end{array}\right) \\
& \text { for } j \equiv_{4} 3: \quad\left(\begin{array}{cccc}
1^{3} & 2^{3} & \cdots & k^{3} \\
1^{7} & 2^{7} & \cdots & k^{7} \\
\vdots & \vdots & \vdots & \vdots \\
1^{4 k-1} & 2^{4 k-1} & \cdots & k^{4 k-1}
\end{array}\right)\left(\begin{array}{c}
2\left|\beta_{1}\right| \sin \left(x+\theta_{1}\right) \\
2\left|\beta_{2}\right| \sin \left(2 x+\theta_{2}\right) \\
\vdots \\
2\left|\beta_{k}\right| \sin \left(k x+\theta_{k}\right)
\end{array}\right)=\left(\begin{array}{c}
0 \\
0 \\
\vdots \\
0
\end{array}\right)
\end{aligned}
$$

Observe that the matrices we obtain are minors of Vandermonde Matrices (up to their sign), and as such are non-singular [9]. It follows that

$$
\left(\begin{array}{c}
2\left|\beta_{1}\right| \sin \left(x+\theta_{1}\right) \\
2\left|\beta_{2}\right| \sin \left(2 x+\theta_{2}\right) \\
\vdots \\
2\left|\beta_{k}\right| \sin \left(k x+\theta_{k}\right)
\end{array}\right)=\left(\begin{array}{c}
0 \\
0 \\
\vdots \\
0
\end{array}\right) \text { and }\left(\begin{array}{c}
2\left|\beta_{1}\right| \cos \left(x+\theta_{1}\right) \\
2\left|\beta_{2}\right| \cos \left(2 x+\theta_{2}\right) \\
\vdots \\
2\left|\beta_{k}\right| \cos \left(k x+\theta_{k}\right)
\end{array}\right)=\left(\begin{array}{c}
0 \\
0 \\
\vdots \\
0
\end{array}\right)
$$

Recall that we assume at least one $\beta_{j^{\prime}}$ is nonzero for some $1 \leq j^{\prime} \leq k$, so we have $\cos \left(j^{\prime} x+\theta_{j^{\prime}}\right)=\sin \left(j^{\prime} x+\theta_{j^{\prime}}\right)=0$, which is clearly a contradiction. We thus conclude the proof.

By Proposition 1, it follows that $g$ is not constant, and therefore $f(x, y)$ is not constant on the curve $x^{2}+y^{2}=1$. By Bezout's Theorem, we have that $Z_{f}$ is finite, and consists of at most $4 k$ points. Moreover, $f$ is a semialgebraic function (that is, its graph $\{(x, y, f(x, y)): x, y \in \mathbb{R}\}$ is semialgebraic set in $\left.\mathbb{R}^{3}\right)$. Thus, the points in $Z_{f}$ have semialgebraic coordinates, and we can compute them. By identifying $\mathbb{R}^{2}$ with $\mathbb{C}$, denote $Z_{f}=\left\{z_{1}, \ldots, z_{4 k}\right\}$.

Remark 2 Since the polynomial $f$ has algebraic coefficients, it is not immediately clear how the degree and height of the points in $Z_{f}$ relate to $\|f\|$. However, recall that the algebraic coefficients in $f$ are polynomials in the entries of $A^{n} s$, which are,

\footnotetext{
4 By splitting modulo 2, we could actually improve the bound in the proposition from $4 k$ to $2 k$, but this further complicates the proof.
} 
in turn, algebraic numbers of degree at most 3 whose description is polynomial in that of $A$ and $s$.

Thus, we can define $Z_{f}$ with a formula in the first-order theory of the reals with a fixed number of variables. Using results of Renegar [21], we show in Appendix D that the points in $Z_{f}$ have semialgebraic coordinates with description length polynomial in $\|f\|$.

We now employ the following lemma from [18], which is itself a consequence of the Baker-Wüstholz Theorem [3].

Lemma 2 ([18]) There exists $D \in \mathbb{N}$ such that for all algebraic numbers $\zeta, \xi$ of

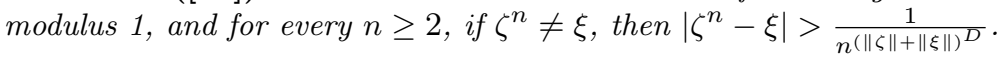

Since $\gamma$ is not a root of unity, it holds that $\gamma^{n_{1}} \neq \gamma^{n_{2}}$ for every $n_{1} \neq n_{2} \in \mathbb{N}$. Thus, there exists a computable $N_{1} \in \mathbb{N}$ such that $\gamma^{n} \notin Z_{f}$ for every $n>N_{1}$. Moreover, by [6, Lemma D.1], we have that $N_{1}=\|f\|^{\mathcal{O}(1)}$. By Lemma 2, there exists a constant $D \in \mathbb{N}$ such that for every $n \geq N_{1}$ and $1 \leq j \leq 4 k$ we have that $\left|\gamma^{n}-z_{j}\right|>\frac{1}{n^{\left(\|f\|^{D}\right)}}\left(\right.$ since $\left.\left\|z_{j}\right\|+\|\gamma\|=\mathcal{O}(\|f\|)\right)$. Intuitively, for $n>N_{1}$ we have that $\gamma^{n}$ does not get close to any $z_{i}$ "too quickly" as a function of $n$. In particular, for $n>N_{1}$ we have $f\left(\gamma^{n}\right) \neq 0$. It thus remains to show that for large enough $n$, $r(n)$ does not affect the sign of $f\left(\gamma^{n}\right)+r(n)$. Intuitively, this is the case because $r(n)$ decreases exponentially, while $\left|f\left(\gamma^{n}\right)\right|$ is bounded from below by an inverse polynomial.

For every $z_{j} \in Z_{f}$, let $\varphi_{j}=\arg z_{j}$, so that $f(z)=0$ iff $g\left(\varphi_{j}\right)=0$. We assume without loss of generality that $\varphi_{j} \in(-\pi, \pi)$ for every $1 \leq j \leq 4 k$. Indeed, if $\varphi_{j}=\pi$ for some $j$, then we can shift the domain of $g$ slightly so that all zeros are in the interior.

For every $1 \leq j \leq 4 k$, let $T_{j}$ be the Taylor polynomial of $g$ around $\varphi_{j}$ such that the degree $d_{j}$ of $T_{j}$ is minimal and $T_{j}$ is not identically 0 . Thus, we have $T_{j}(x)=\frac{g^{\left(d_{j}\right)}\left(\varphi_{j}\right)}{d_{j} !}\left(x-\varphi_{j}\right)^{d_{j}}$. By Proposition 1 we have that $d_{j} \leq 4 k$ for every $j$. In addition, the description of $T_{j}$ is computable from that of $\|f\|$.

By Taylor's inequality, we have that for every $x \in[-\pi, \pi]$ it holds that $\mid g(x)-$ $T_{j}(x) \mid \leq \frac{M_{j}\left|x-\varphi_{j}\right|^{d_{j}+1}}{\left(d_{j}+1\right) !}$ where $M_{j}=\max _{x \in[-\pi, \pi]}\left\{g^{\left(d_{j}+1\right)}(x)\right\}$ (where $g$ is extended naturally to the domain $[-\pi, \pi])$. By our description of $g^{\left(d_{j}+1\right)}(x)$, we see that $M_{j}$ is bounded by $M=4 k \max _{1 \leq i \leq k}\left\{\left|\beta_{i}\right|\right\} k^{4 k+1}$.

Let $\epsilon_{1}>0$ be such that the following conditions hold for every $1 \leq j \leq 4 k$.

1. $\operatorname{sign}\left(g^{\prime}(x)\right)$ does not change in $\left(\varphi_{j}, \varphi_{j}+\epsilon_{1}\right)$ nor in $\left(\varphi_{j}-\epsilon_{1}, \varphi_{j}\right)$.

2. $\left|g(x)-T_{j}(x)\right| \leq \frac{1}{2}\left|T_{j}(x)\right|$ for every $x \in\left(\varphi_{j}-\epsilon_{1}, \varphi_{j}+\epsilon_{1}\right)$.

3. $\operatorname{sign}\left(g^{\prime}(x)\right)=\operatorname{sign}\left(T_{j}^{\prime}(x)\right)$ for every $x \in\left(\varphi_{j}-\epsilon_{1}, \varphi_{j}+\epsilon_{1}\right)$.

Note that we can assume $\left(\varphi_{j}-\epsilon_{1}, \varphi_{j}+\epsilon_{1}\right) \subseteq(-\pi, \pi)$, since by our assumption $\varphi_{j} \in(-\pi, \pi)$ for all $1 \leq j \leq 4 k$.

An $\epsilon_{1}$ as above exists due to the following properties (see Figure 1 for an illustration):

- There are only finitely many points where $g^{\prime}(x)=0$,

- $T_{j}(x)$ is of degree $d_{j}$, whereas $\left|g(x)-T_{j}(x)\right|$ is upper-bounded by a polynomial of degree $d_{j}+1$, and 


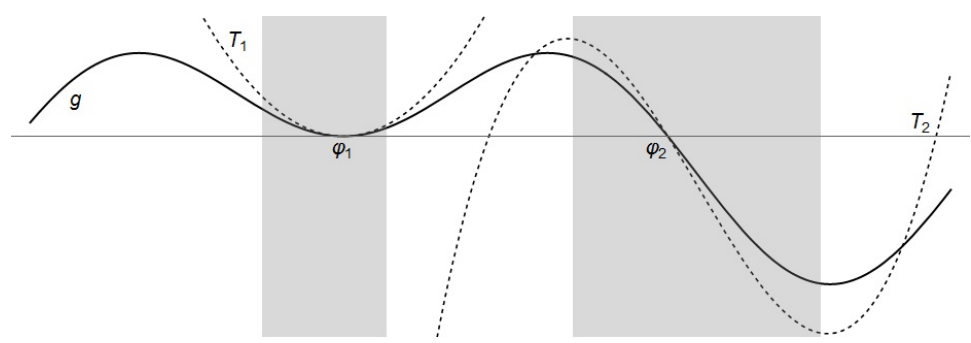

Fig. $1 g(x)$ and two Taylor polynomials: $T_{1}(x)$ around $\varphi_{1}$ and $T_{2}(x)$ around $\varphi_{2}$. The shaded regions show where requirements (1)-(3) hold, which determine $\epsilon_{1}$. Observe that for $T_{1}$, the most restrictive requirement is $\left|g(x)-T_{1}(x)\right| \leq \frac{1}{2} T_{1}(x)$, whereas for $T_{2}$ the restriction is the requirement that $T_{2}(x)$ is monotone.

- $T_{j}^{\prime}(x)$ is the Taylor polynomial of degree $d_{j}-1$ of $g^{\prime}(x)$ around $\varphi_{j}$, so by bounding the distance $\left|g^{\prime}(x)-T_{j}^{\prime}(x)\right|$ we can conclude the third requirement.

In order to establish Lemma 1 , we must be able to effectively compute $\epsilon_{1}$. We thus proceed with the following lemma.

Lemma $3 \epsilon_{1}$ can be computed in polynomial time in $\|f\|$, and $\frac{1}{\epsilon_{1}}=2^{\|f\|^{\mathcal{O}(1)}}$.

Proof We compute $\delta_{1}, \delta_{2}, \delta_{3}$ that satisfy requirements 1,2 , and 3 , respectively. Then, taking $\epsilon_{1}=\min \left\{\delta_{1}, \delta_{2}, \delta_{3}\right\}$ will conclude the proof.

Condition 1: We compute $\delta_{1}>0$ such that $\operatorname{sign}\left(g^{\prime}(x)\right)$ does not change in $\left(\varphi_{j}-\right.$ $\left.\delta_{1}, \varphi_{j}\right)$ nor in $\left(\varphi_{j}, \varphi_{j}+\delta_{1}\right)$. This is done as follows. Recall that $g(x)=f\left(e^{i x}\right)=$ $\sum_{m=0}^{k} \beta_{m} e^{i m x}+\overline{\beta_{m}} e^{i m x}$. It is not hard to check that $g^{\prime}(x)=\sum_{m=0}^{k} i m \beta_{m} e^{i m x}+$ $\overline{i m \beta_{m}} e^{i m x}$. Let $\widehat{f}(z): \mathbb{C} \rightarrow \mathbb{R}$ be the function $\widehat{f}(z)=\sum_{m=0}^{k} i m \beta_{m} z+\overline{i m \beta_{m}} \bar{z}$, then $g^{\prime}(x)=\widehat{f}\left(e^{i x}\right)$ and $\|\widehat{f}\|=\mathcal{O}(\|f\|)$.

Consider the algebraic set $F=\{z:|z|=1 \wedge \widehat{f}(z)=0\}$, then $\left\{x: g^{\prime}(x)=0\right\}=$ $\{\arg (z): z \in F\}$. By similar arguments as those by which we found the roots of $f$ on the unit circle, namely by adapting Proposition 1 to $\widehat{f}$, we can conclude that $F$ contains at most $4 k$ points. Thus, it is enough to set $\delta_{1}$ such that $\left(\bigcup_{j=1}^{4 k}\left(\varphi_{j}-\delta_{1}, \varphi_{j}\right) \cup\left(\varphi_{j}, \varphi_{j}+\delta_{1}\right)\right) \cap F=\emptyset$.

By Equation (1), we have that for $z \neq z^{\prime} \in F$ it holds that $\left|z-z^{\prime}\right|>\frac{\sqrt{6}}{d^{\frac{d+1}{2}} \cdot H^{d-1}}$ where $d$ and $H$ are the degree and height of the roots of $\widehat{f}(z)$ (see Remark 2). Thus, $1 /\left|z-z^{\prime}\right|$ is $2^{\|f\|^{\mathcal{O}(1)}}$, and has a polynomial description. Since $\left|\arg (z)-\arg \left(z^{\prime}\right)\right|>$ $\left|z-z^{\prime}\right|$, we conclude that by setting $\delta_{1}=\min \left\{\left|z-z^{\prime}\right|: z \neq z^{\prime} \in F\right\} / 3$, it holds that $\frac{1}{\delta_{1}}$ has a polynomial description in $\|f\|$, and $\delta_{1}$ satisfies the required condition.

Condition 2: Next, we compute $\delta_{2}>0$ such that $\left|g(x)-T_{j}(x)\right| \leq \frac{1}{2}\left|T_{j}(x)\right|$ for every $x \in\left(\varphi_{j}-\delta_{2}, \varphi_{j}+\delta_{2}\right)$. Recall that $T_{j}(x)=\frac{g^{\left(d_{j}\right)}\left(\varphi_{j}\right)}{d_{j} !}\left(x-\varphi_{j}\right)^{d_{j}}$. Note that this case is more challenging than Condition 1 , as unlike $g(x)=f\left(e^{i x}\right)$, the polynomial $T_{j}(x)$ has potentially transcendental coefficients (namely $\varphi_{j}$ ). For clarity, we omit the index $j$ in the following. Thus, we write $T, d, \varphi$ instead of $T_{j}, d_{j}, \varphi_{j}$, etc. 
In order to ignore the absolute value, assume $T(x) \geq g(x)>0$ in an interval $(\varphi, \varphi+\xi)$ for some $\xi>0$ (the other cases are treated similarly). Then, the inequality above becomes $g(x)-\frac{1}{2} T(x) \geq 0$. Since the degree of $T$ is $d$, then by the definition of $T$, the first $d-1$ derivatives of $g$ in $\varphi$ vanish. Define $h(x)=g(x)-\frac{1}{2} T(x)$, then we have $h(\varphi)=0, h^{\prime}(\varphi)=0, \ldots, h^{(d-1)}(\varphi)=0$ and $h^{(d)}(\varphi)=g^{(d)}(\varphi)-\frac{1}{2} g^{(d)}(\varphi)=\frac{1}{2} g^{(d)}(\varphi)$. By our assumption, $T(x) \geq \frac{1}{2} T(x)$ for $x \in(\varphi, \varphi+\xi)$, so $h^{(d)}(\varphi)>0$. In addition, recall that $\left|h^{(d+1)}(x)\right|=\left|g^{(d+1)}(x)\right| \leq M$ for every $x \in[-\pi, \pi]$. Thus, by writing the $d$-th Taylor expansion of $h(x)$ around $\varphi$, we have that $h(x)=\frac{h^{(d)}(\varphi)}{d !}(x-\varphi)^{d}+E(x)$ where $|E(x)| \leq \frac{M}{(d+1) !}(x-\varphi)^{d+1}$. We now have that

$$
h(x) \geq \frac{1}{2} \frac{g^{(d)}(\varphi)}{d !}(x-\varphi)^{d}-\frac{M}{(d+1) !}(x-\varphi)^{d+1} .
$$

Taking $x \in\left(\varphi, \varphi+\frac{g^{(d)}(\varphi)(d+1)}{2 M}\right)$, it is easy to check that $h(x) \geq 0$. We can now set $\delta_{2}=\frac{g^{(d)}(\varphi)(d+1)}{2 M}$, which satisfies the required condition.

Condition 3: Finally, we compute $\delta_{3}>0$ such that $\operatorname{sign}\left(g^{\prime}(x)\right)=\operatorname{sign}\left(T_{j}^{\prime}(x)\right)$ for every $x \in\left(\varphi_{j}-\delta_{3}, \varphi_{j}+\delta_{3}\right)$. Observe that $T_{j}^{\prime}(x)$ is the $\left(d_{j}-1\right)$-th Taylor polynomial of $g^{\prime}(x)$ around $\varphi^{j}$. Thus, by following the reasoning used to find $\delta_{2}$, we can find $\delta_{3}$ such that $\left|g^{\prime}(x)-T_{j}^{\prime}(x)\right| \leq \frac{1}{2}\left|T_{j}^{\prime}(x)\right|$ for every $x \in\left(\varphi-\delta_{3}, \varphi+\delta_{3}\right)$, and in particular it holds that $\operatorname{sign}\left(g^{\prime}(x)\right)=\operatorname{sign}\left(T_{j}^{\prime}(x)\right)$ for every $x \in\left(\varphi_{j}-\delta_{3}, \varphi_{j}+\delta_{3}\right)$.

As mentioned above, by setting $\epsilon_{1}=\min \left\{\delta_{1}, \delta_{2}, \delta_{3}\right\}$, we conclude the proof.

Conditions 1,2 , and 3 above imply that within the intervals $\left(\varphi_{j}-\epsilon_{1}, \varphi_{j}+\epsilon_{1}\right)$ we have that $|g(x)| \geq \frac{1}{2}\left|T_{j}(x)\right|$, that $g(x)$ and $T_{j}(x)$ have the same sign, and that they are both decreasing/increasing together.

We now claim that there exist a polynomial $p(n)$ and a number $N_{2} \in \mathbb{N}$ such that for every $n>N_{2}$ it holds that $\left|g\left(\arg \left(\gamma^{n}\right)\right)\right|>\frac{1}{p(n)}$. In order to compute $p(n)$, we compute separate polynomials for the domain $\bigcup_{j=1}^{4 k}\left(\varphi_{j}-\epsilon_{1}, \varphi_{j}+\epsilon_{1}\right)$ and for its complement. Then, taking their minimum and bounding it from below by another polynomial yields $p(n)$.

We start by considering the case where $\arg \left(\gamma^{n}\right) \in \bigcup_{j=1}^{4 k}\left(\varphi_{j}-\epsilon_{1}, \varphi_{j}+\epsilon_{1}\right)$. Recall that since $\gamma$ is not a root of unity, then for every $n>N_{1}$ it holds that $\gamma^{n} \notin$ $Z_{f}=\left\{z_{1}, \ldots, z_{4 k}\right\}$. Then, by Lemma 2 , for every $1 \leq j \leq 4 k$ and every $n \geq N_{2}=$ $\max \left\{N_{1}, 2\right\}$ we have $\left|\gamma^{n}-z_{j}\right|>\frac{1}{n^{\left(\|f\|^{D}\right)}}$. In addition, $\left|\gamma^{n}-z_{j}\right| \leq\left|\arg \left(\gamma^{n}\right)-\varphi_{j}\right|$ (since the LHS is the Euclidean distance and the RHS is the spherical distance). Therefore, $\left|\arg \left(\gamma^{n}\right)-\varphi_{j}\right|>\frac{1}{n^{\left(\|f\|^{D}\right)}}$, so either $\arg \left(\gamma^{n}\right)>\varphi_{j}+\frac{1}{n^{\left(\|f\|^{D}\right)}}$ or $\arg \left(\gamma^{n}\right)<\varphi_{j}-\frac{1}{n^{\left(\|f\|^{D}\right)}}$. Next, we have that if $\arg \left(\gamma^{n}\right) \in\left(\varphi_{j}-\epsilon_{1}, \varphi_{j}+\epsilon_{1}\right)$ for some $1 \leq j \leq 4 k$, then $\left|g\left(\arg \left(\gamma^{n}\right)\right)\right| \geq \frac{1}{2}\left|T_{j}\left(\arg \left(\gamma^{n}\right)\right)\right| \geq \frac{1}{2} \min \left\{\left|T_{j}\left(\varphi_{j}+\frac{1}{n^{\left(\|f\|^{D}\right)}}\right)\right|,\left|T_{j}\left(\varphi_{j}-\frac{1}{n^{\left(\|f\|^{D}\right)}}\right)\right|\right\}$, where the last inequality follows from condition 3 above, which implies that $T_{j}$ is monotone with the same tendency as $g$.

Observe that $T_{j}\left(\varphi_{j}-\frac{1}{n^{\left(\|f\|^{D}\right)}}\right)=\frac{g^{\left(d_{j}\right)}(\varphi)}{d_{j} !} \frac{1}{n^{\left(\|f\|^{D}\right)}}$ and that similarly $T_{j}\left(\varphi_{j}+\right.$ $\left.\frac{1}{n^{\left(\|f\|^{D}\right)}}\right)=-\frac{g^{\left(d_{j}\right)}(\varphi)}{d_{j} !} \frac{1}{n^{\left(\|f\|^{D}\right)}}$ are both inverse polynomials (in $\left.n\right)$. Thus, $\left|g\left(\arg \left(\gamma^{n}\right)\right)\right|$ is bounded from below by an inverse polynomial. Moreover, these polynomials can be easily computed in time polynomial in $\|f\|$. 
Finally, we note that for $x \notin \bigcup_{j=1}^{4 k}\left(\varphi_{j}-\epsilon_{1}, \varphi_{j}+\epsilon_{1}\right)$ we can compute in polynomial time a bound $B>0$ such that $|g(x)|>B$. Indeed, $B=\min \{|g(x)|: x \in[-\pi, \pi] \backslash$ $\left.\bigcup_{j=1}^{4 k}\left(\varphi_{j}-\epsilon_{1}, \varphi_{j}+\epsilon_{1}\right)\right\}$ (where $g(-\pi)$ is defined naturally by extending the domain), and we have that $|B|>0$ since we assumed none of the $\varphi_{j}$ are exactly at $\pi$ (in which case we would have had $g(-\pi)=0$ ). In particular, we can combine the two domains and compute a polynomial $p$ as required. We remark that we can compute $\|B\|$ in polynomial time, since it is either at least $\frac{1}{2}\left|T_{j}\left(\varphi_{j} \pm \epsilon_{1}\right)\right|$ for some $1 \leq j \leq 4 k$ (and by Lemma $3,\left\|\epsilon_{1}\right\|$ can be computed in polynomial time), or it is the value of one of the extrema of $g$, and the latter can be computed by finding the extrema of the (algebraic) function $f$ on the unit circle.

To recap, for every $n>N_{2}$ it holds that $\left|g\left(\arg \left(\gamma^{n}\right)\right)\right|>\frac{1}{p(n)}$ for a non-negative polynomial $p$, and both $N_{2}$ and $p$ can be computed in polynomial time in the description of the input.

Next, we wish to find $N_{3} \in \mathbb{N}$ such that for every $n>N_{3}$ it holds that $r(n)<\frac{1}{p(n)}$. Recall that $r(n)=\sum_{l=1}^{k^{\prime}} \chi_{l} \mu_{l}^{n}+{\overline{\chi_{l} \mu_{l}}}^{n}$ where for every $1 \leq l \leq k^{\prime}$ we have that $\mu_{l}$ is algebraic with $\operatorname{deg}\left(\mu_{l}\right)=\|f\|^{\mathcal{O}(1)}$ and $H\left(\mu_{l}\right)=2^{\|f\|^{\mathcal{O}(1)}}$. Observe that $1-\left|\mu_{l}\right|$ is also an algebraic number. Indeed, $1-\left|\mu_{l}\right|=1-\sqrt{\mu_{l} \overline{\mu_{l}}}$. Moreover, we get that $\operatorname{deg}\left(1-\left|\mu_{l}\right|\right) \leq \operatorname{deg}\left(\mu_{l}\right)^{4}$, as it is the root of a polynomial of degree at most $\operatorname{deg}\left(\mu_{l}\right)^{4}$, and that $H\left(1-\left|\mu_{l}\right|\right)$ is polynomial in $H\left(\mu_{l}\right)$. Since $\left|\mu_{l}\right|<1$, by applying Equation (1), we get $1-\left|\mu_{l}\right|=|1-| \mu_{l}||>\frac{\sqrt{6}}{d^{(d+1) / 2} H\left(\mu_{l}\right)^{d-1}}$ where $d=\operatorname{deg}\left(\mu_{l}\right)^{\mathcal{O}(1)}$ and $H\left(\mu_{l}\right)=2^{\|I\|^{\mathcal{O}(1)}}$. It follows that we can compute $\delta \in(0,1)$ with $\frac{1}{\delta}=2^{\|I\|^{\mathcal{O}(1)}}$ such that $1-\left|\mu_{l}\right|>\delta$, and hence $|\mu|^{n}<1-\delta$. Thus,

$$
|r(n)| \leq \sum_{l=1}^{k^{\prime}} 2\left|\chi_{l}\right|\left|\mu_{l}\right|^{m n} \leq \sum_{l=1}^{k^{\prime}} 2\left|\chi_{l}\right|(1-\delta)^{m n} \leq 2 k^{\prime} \max _{1 \leq l \leq k^{\prime}}\left|\chi_{l}\right|(1-\delta)^{n}
$$

We can now compute $\epsilon \in(0,1)$ and $N_{3} \in \mathbb{N}$ such that:

1. $\frac{1}{\epsilon}=2^{\|I\|^{\mathcal{O}(1)}}$

2. $N_{3}=2^{\|I\|^{\mathcal{O}(1)}}$

3. For every $n>N_{3}$ it holds that $|r(n)|<(1-\epsilon)^{n}$

Finally, by taking $N_{4} \in \mathbb{N}$ such that $(1-\epsilon)^{n}<\frac{1}{p(n)}$ (which satisfies $N_{4}=$ $2^{\|I\|^{\mathcal{O}(1)}}$ ) for all $n>N_{4}$, we can now conclude that for every $n>\max \left\{N_{2}, N_{3}, N_{4}\right\}$, the following hold.

1. $f\left(\gamma^{n}\right)=g\left(\arg \left(\gamma^{n}\right)\right) \neq 0$.

2. If $f\left(\gamma^{n}\right)>0$, then $g\left(\arg \left(\gamma^{n}\right)\right)>0$, so $g\left(\arg \left(\gamma^{n}\right)\right)>\frac{1}{p(n)}$. Since $|r(n)|<\frac{1}{p(n)}$, it follows that $f\left(\gamma^{n}\right)+r(n)=g\left(\arg \left(\gamma^{n}\right)\right)+r(n)>\frac{1}{p(n)}-|r(n)|>0$. Conversely, if $f\left(\gamma^{n}\right)+r(n)>0$, then $g\left(\arg \left(\gamma^{n}\right)\right)+r(n)>0$, but since $\left|g\left(\arg \left(\gamma^{n}\right)\right)\right|>\frac{1}{p(n)}$ and $|r(n)|<\frac{1}{p(n)}$, then it must hold that $g\left(\arg \left(\gamma^{n}\right)\right)>0$, so $f\left(\gamma^{n}\right)>0$.

3. If $f\left(\gamma^{n}\right)<0$, then $g\left(\arg \left(\gamma^{n}\right)\right)<0$, so $g\left(\arg \left(\gamma^{n}\right)\right)<-\frac{1}{p(n)}$. Since $|r(n)|<\frac{1}{p(n)}$, it follows that $f\left(\gamma^{n}\right)+r(n)=g\left(\arg \left(\gamma^{n}\right)\right)+r(n)<-\frac{1}{p(n)}+|r(n)|<0$. Conversely, if $f\left(\gamma^{n}\right)+r(n)<0$, then $g\left(\arg \left(\gamma^{n}\right)\right)+r(n)<0$, but since $\left|g\left(\arg \left(\gamma^{n}\right)\right)\right|>\frac{1}{p(n)}$ and $|r(n)|<\frac{1}{p(n)}$, then it must hold that $g\left(\arg \left(\gamma^{n}\right)\right)<0$, so $f\left(\gamma^{n}\right)<0$.

This concludes the proof of Lemma 1.

We are now ready to use Lemma 1 in order to solve the systems. 
Theorem 4 The problem of deciding whether an almost self-conjugate system has a solution is decidable.

Proof Consider an almost self-conjugate system of the form $\bigwedge_{J} R_{J}\left(A^{n} \vec{s}\right) \sim_{J} 0$. For each expression $R_{J}\left(A^{n} \vec{s}\right) \sim_{J} 0$, let $f$ be the corresponding dominant function, as per Lemma 1, and compute its respective bound $N$. If $\sim_{J}$ is "=", then by Lemma 1 , if the equation is satisfiable for $n \in \mathbb{N}$, then $n<N$.

If all the $\sim_{J}$ are ">", then for each such inequality compute $\{z: f(z)>0\}$, which is a semialgebraic set. If the intersection of these sets is empty, then if $n$ is a solution for the system, it must hold that $n<N$. If the intersection is non-empty, then it is an open set. Since $\gamma$ is not a root of unity, then $\left\{\gamma^{n}: n \in \mathbb{N}\right\}$ is dense in the unit circle. Thus, there exists $n>N$ such that $\gamma^{n}$ is in the above intersection, so the system has a solution. Checking the emptiness of the intersection can be done using Theorem 1.

Thus, it remains to check whether there exists a solution $n<N$, which is clearly decidable.

Observe that from Theorem 4, combined with Section 4.1, we can conclude the decidability of quantifier-free FOOQ Problem. However, as it turns out, we can reuse Theorem 4 to obtain a much stronger result, namely the decidability of the general Fooq Problem.

\section{Quantifier Elimination from First-Order Orbit Queries}

In this section we complete the proof of Theorem 3, showing that the FOOQ Problem is decidable. Intuitively, this is done by reducing the general problem to the quantifier-free case of Section 4 . Technically, we do not actually construct an equivalent quantifier-free query, but rather reduce the FOOQ Problem to the problem of solving a system of almost self-conjugate polynomials, and then apply Theorem 4.

Proof (of Theorem 3) Consider a matrix $A \in \mathbb{Q}^{3 \times 3}$ and let $\Phi(n)=Q_{1} x_{1}, \ldots, Q_{m} x_{m}$ $\Psi\left(n, x_{1}, \ldots, x_{m}\right)$ be a FOOQ as described in Section 3 . We again assume that $A$ has a complex eigenvalue. As in Section 4, the case where all eigenvalues are real is handled in Appendix A.

Consider an orbit proposition $A^{n} \vec{x} \in T$ in $\Psi$, where $\vec{x}=\left(x_{1}, x_{2}, x_{3}\right)$ consists of (possibly) quantified variables. ${ }^{5}$ Following a similar analysis to Section 4.1, we write $A=P D P^{-1}$ with $D=\left(\begin{array}{ccc}\lambda & 0 & 0 \\ 0 & \lambda & 0 \\ 0 & 0 & \rho\end{array}\right)$, where $\lambda$ is a complex eigenvalue, $\rho \in \mathbb{R}$, and

$P$ an invertible matrix. Then, we have $A^{n} \vec{x}=P D^{n} P^{-1} \vec{x}$, and for every $1 \leq i \leq 3$ we have $A^{n}(\vec{x})_{i}=\sum_{j=1}^{3}\left(a_{i, j} \lambda^{n}+\bar{a}_{i, j} \bar{\lambda}^{n}+b_{i, j} \rho^{n}\right) x_{j}$ with $a_{i, j} \in \mathbb{A}$ and $b_{i, j} \in \mathbb{A} \cap \mathbb{R}$. That is, each coordinate $1 \leq i \leq 3$, is a linear combination of $x_{1}, x_{2}, x_{3}$ where the coefficients are of the form above. In particular, the coefficient of every $x_{j}$ is an almost self-conjugate polynomial (see Appendix $\mathrm{C}$ for a complete analysis).

Next, again as in Section 4.1, we write $T=\left\{(x, y, z): \bigvee_{I} \bigwedge_{J} R_{I, J}(x, y, z) \sim_{I, J} 0\right\}$ where $R_{I, J}$ are polynomials with integer coefficients, and $\sim_{I, J} \in\{>,=\}$.

5 We use $x_{1}, x_{2}, x_{3}$ to represent constants or variables from $x_{1}, \ldots, x_{m}$. For readability, we do not introduce double indices for these variables. 
Consider a monomial of the form $x_{1}^{s_{1}} x_{2}^{s_{2}} x_{3}^{s_{3}}$ in $R_{I, J}(\vec{x})$. Replacing $\vec{x}$ with $A^{n} \vec{x}$, the monomial then becomes $Q\left(\lambda^{n}, \bar{\lambda}^{n}, \rho^{n}\right) x_{1}^{s_{1}} x_{2}^{s_{2}} x_{3}^{s_{3}}$, where $Q\left(z_{1}, z_{2}, z_{3}\right)$ is an almost self-conjugate polynomial. Indeed, this follows since the coordinates of $A^{n} \vec{x}$ above are almost self-conjugate polynomials, and products of almost self-conjugate polynomials remain almost self-conjugate.

Recall that the polynomials $R_{I, J}$ in the description of $T$ have integer (and in particular, real) coefficients. By lifting the discussion about monomials to $R_{I, J}$, we can write

$$
R_{I, J}\left(A^{n}(\vec{x})\right)=\sum_{0 \leq s_{1}, s_{2}, s_{3} \leq k} Q_{s_{1}, s_{2}, s_{3}}^{I, J}\left(\lambda^{n}, \bar{\lambda}^{n}, \rho^{n}\right) x_{1}^{s_{1}} x_{2}^{s_{2}} x_{3}^{s_{3}}
$$

where $k \in \mathbb{N}$ and the coefficients $Q_{s_{1}, s_{2}, s_{3}}^{I, J}$ are almost self-conjugate.

Thus, we can replace the orbit proposition $A^{n} \vec{x} \in T$ with $\bigvee_{I} \bigwedge_{J} R_{I, J}\left(A^{n}(\vec{x})\right) \sim_{I, J}$ 0 , with $R_{I, J}\left(A^{n}(\vec{x})\right)$ expanded as above.

Applying this to every orbit propositions in $\Psi$ and converting $\Psi$ to DNF, we can write $\Psi$ as

$$
\bigvee_{E} \bigwedge_{F} S_{E, F}\left(x_{1}^{E, F}, x_{2}^{E, F}, x_{3}^{E, F}\right) \sim_{E, F, 1} 0 \wedge g^{E, F}\left(x_{1}, \ldots, x_{m}\right) \sim_{E, F, 2} 0
$$

where $g^{E, F} \in \mathbb{Z}[\vec{x}], \sim_{E, F, 1}, \sim_{E, F, 2} \in\{>,=\}, x_{i}^{E, F} \in\left\{x_{1}, \ldots, x_{m}\right\}$ for every $i \in$ $\{1,2,3\}$, and

$$
S^{E, F}(x, y, z)=\sum_{0 \leq s_{1}, s_{2}, s_{3} \leq k} Q_{s_{1}, s_{2}, s_{3}}^{E, F}\left(\lambda^{n}, \bar{\lambda}^{n}, \rho^{n}\right) x^{s_{1}} y^{s_{2}} z^{s_{3}}
$$

Note that we must include the "standard" first-order atomic propositions $g^{E, F}$ in every conjunct, which leads to slightly cumbersome indexing.

Intuitively, we now want to eliminate the quantifiers on $x_{1}, \ldots, x_{m}$ in the expression above. However, we cannot readily do so, as the expression is also quantified by $n \in \mathbb{N}$. Nonetheless, in the following we manage to circumvent this problem by increasing the dimension of the problem.

Let $K$ be the number of polynomials $Q_{s_{1}, s_{2}, s_{3}}^{E, F}$ that appear in $\Psi$, indexed by $E, F, s_{1}, s_{2}, s_{3}$. We introduce real-valued variables $y_{s_{1}, s_{2}, s_{3}}^{E, F}$ that, intuitively, serve as placeholders for the polynomials $Q_{s_{1}, s_{2}, s_{3}}^{E, F}\left(\lambda^{n}, \bar{\lambda}^{n}, \rho^{n}\right)$. Thus, define $\widetilde{S}^{E, F}(x, y, z)=$ $\sum_{0 \leq s_{1}, s_{2}, s_{3} \leq k} y_{s_{1}, s_{2}, s_{3}}^{E, F} x^{s_{1}} y^{s_{2}} z^{s_{3}}$.

Consider the set

$$
U=\left\{\left(y_{1}, \ldots, y_{K}\right) \in \mathbb{R}^{K}: \begin{array}{l}
Q_{1} x_{1}, \ldots Q_{m} x_{m}, \bigvee_{E} \bigwedge_{F} \\
\widetilde{S}_{E, F}\left(x_{1}^{E, F}, x_{2}^{E, F}, x_{3}^{E, F}\right) \sim_{E, F, 1} 0 \\
\wedge g^{E, F}\left(x_{1}, \ldots, x_{m}\right) \sim_{E, F, 2} 0
\end{array}\right\}
$$

Observe that $U$ is a semialgebraic set. Indeed, the terms in $\widetilde{S}_{E, F}$ are not dependent on $S$, and so these are polynomials. Thus, by Theorem 2 , we can eliminate the quantifiers on $x_{1}, \ldots, x_{m}$, and write

$$
U=\left\{\left(y_{1}, \ldots, y_{K}\right) \in \mathbb{R}^{K}: \bigvee_{I} \bigwedge_{J} V_{I, J}\left(y_{1}, \ldots, y_{K}\right) \sim_{I, J} 0\right\}
$$


where $V_{I, J}$ are polynomials with integer coefficients (and we re-use the indices $I, J)$. It is now the case that $\exists n \in \mathbb{N} \Phi(n)$ holds iff there exists $n \in \mathbb{N}$ such that $\left(Q_{1}\left(\lambda^{n}, \bar{\lambda}^{n}, \rho^{n}\right), \ldots, Q_{K}\left(\lambda^{n}, \bar{\lambda}^{n}, \rho^{n}\right)\right) \in U$. That is, we need to decide whether there exist $I$ and $n \in \mathbb{N}$ such that $V_{I, J}\left(Q_{1}\left(\lambda^{n}, \bar{\lambda}^{n}, \rho^{n}\right), \ldots, Q_{K}\left(\lambda^{n}, \bar{\lambda}^{n}, \rho^{n}\right)\right) \sim_{I, J} 0$ for every $J$.

It is easy to see that since the polynomials $Q_{s_{1}, s_{2}, s_{3}}^{E, F}$ are almost self-conjugate, then so is $V_{I, J}\left(Q_{1}\left(\lambda^{n}, \bar{\lambda}^{n}, \rho^{n}\right), \ldots, Q_{K}\left(\lambda^{n}, \bar{\lambda}^{n}, \rho^{n}\right)\right.$ (when viewed as a polynomial in $\left.\lambda^{n}, \bar{\lambda}^{n}, \rho^{n}\right)$.

Thus, the conjunction

$$
\bigwedge_{J} V_{I, J}\left(Q_{1}\left(\zeta^{n}, \bar{\zeta}^{n}, \eta^{n}\right), \ldots, Q_{K}\left(\zeta^{n}, \bar{\zeta}^{n}, \eta^{n}\right)\right)
$$

is an almost self-conjugate system for every $I$, and by Theorem 4 , it is decidable whether it has a solution. This concludes the proof.

\section{Discussion and Generalizations}

This paper establishes the decidability of First-Order Orbit Queries in dimension at most three. The class of first-order definable sets is arguably the largest natural class for which membership is decidable. Thus, our results reach the limit of what can be decided about the orbit of a single matrix. Moreover, our techniques shed light on the decidability (or hardness) of orbit problems in higher dimensions: the techniques we develop for analysing orbits can be applied to any matrix (in any dimension) whose eigenvalues have arguments that are pairwise linearly dependent over $\mathbb{Q}$ (i.e., the arguments of all the eigenvalues are rational multiples of some angle $\theta$ ). Indeed, it is easy to see that the orbits generated by such matrices can be reduced to solving almost self-conjugate systems (see Section 4). This can be put in contrast to known hardness results [5] in dimension $d \geq 4$, which require a single pair of eigenvalues whose arguments do not satisfy the above property. Thus, we significantly sharpen the border of known decidability, and allow future research to focus on hard instances.

Technically, our contribution uncovers two interesting tools. First, the identification of almost self-conjugate polynomials, and their amenability to analysis (Section 4), and second, the ability to abstract away integral exponents in order to perform quantifier elimination, by increasing the dimension (Section 5). The former arises naturally in the context of matrix exponentiation, while the latter is an obstacle that is often encountered when quantifying over semialgebraic sets in the presence of a discrete operator (e.g., matrix exponentiation). In the future, we plan to further investigate the applications of these directions.

In addition, we remark that our formalism can be slightly generalized, to capture an even wider set of queries: observe that our queries separate orbit propositions from standard first-order proposition. Technically, however, all we require is that the polynomials we work with are almost self-conjugate. Thus, we can also handle queries whose atomic propositions are of the form $g(\vec{x}) \sim 0$ where $g \in \mathbb{Z}[\vec{x}], \sim \in\{>,=\}$, and each entry of $\vec{x}$ is either a real-valued variable, or a coordinate of $A^{n} y$ for a variable $y$. That is, we can combine entries of $A^{n} y$ with first-order variables, within the same atomic proposition. For the sake of clarity, we did not use this formalism in the paper. 


\section{References}

1. S. Almagor, J. Ouaknine, and J. Worrell. The polytope-collision problem. In 44th International Colloquium on Automata, Languages, and Programming, ICALP 2017, July 10-14, 2017, Warsaw, Poland, pages 24:1-24:14, 2017.

2. Shaull Almagor, Joël Ouaknine, and James Worrell. The semialgebraic orbit problem. In 36th International Symposium on Theoretical Aspects of Computer Science, STACS 2019, March 13-16, 2019, Berlin, Germany, pages 6:1-6:15, 2019.

3. A. Baker and G. Wüstholz. Logarithmic forms and group varieties. J. reine angew. Math, 442(19-62):3, 1993.

4. S. Basu, R. Pollack, and M-F. Roy. Algorithms in real algebraic geometry, volume 20033. Springer, 2005.

5. V. Chonev, J. Ouaknine, and J. Worrell. The polyhedron-hitting problem. In Proceedings of the Twenty-Sixth Annual ACM-SIAM Symposium on Discrete Algorithms, pages 940-956. SIAM, 2015.

6. V. Chonev, J. Ouaknine, and J. Worrell. On the complexity of the orbit problem. J. ACM, 63(3):23:1-23:18, 2016.

7. H. Cohen. A course in computational algebraic number theory, volume 138. Springer Science \& Business Media, 2013.

8. G. E. Collins. Quantifier elimination for real closed fields by cylindrical algebraic decompostion. In Automata Theory and Formal Languages 2nd GI Conference Kaiserslautern, May 20-23, 1975, pages 134-183. Springer, 1975.

9. F.R. Gantmacher. The Theory of Matrices. Number v. 2 in The Theory of Matrices. Chelsea Publishing Company, 1959.

10. V. Halava, T. Harju, M. Hirvensalo, and J. Karhumäki. Skolem's problem - on the border between decidability and undecidability. Technical Report 683, Turku Centre for Computer Science, 2005.

11. M. A Harrison. Lectures on linear sequential machines. Technical report, DTIC Document, 1969.

12. R. Kannan and R. J. Lipton. The orbit problem is decidable. In Proceedings of the twelfth annual ACM symposium on Theory of computing, pages 252-261. ACM, 1980.

13. R. Kannan and R. J. Lipton. Polynomial-time algorithm for the orbit problem. Journal of the ACM (JACM), 33(4):808-821, 1986.

14. G. Lafferriere, G. J. Pappas, and S. Yovine. Symbolic reachability computation for families of linear vector fields. J. Symb. Comput., 32(3):231-253, 2001.

15. M. Mignotte. Some useful bounds. In Computer algebra, pages 259-263. Springer, 1983.

16. M. Mignotte, T. Shorey, and R. Tijdeman. The distance between terms of an algebraic recurrence sequence. J. für die reine und angewandte Math., 349, 1984.

17. M. Müller-Olm and H. Seidl. Computing polynomial program invariants. Inf. Process. Lett., 91(5):233-244, 2004.

18. J. Ouaknine and J. Worrell. Ultimate positivity is decidable for simple linear recurrence sequences. In International Colloquium on Automata, Languages, and Programming, pages 330-341. Springer, 2014.

19. V. Y. Pan. Optimal and nearly optimal algorithms for approximating polynomial zeros. Computers \& Mathematics with Applications, 31(12):97-138, 1996.

20. J. Renegar. A faster PSPACE algorithm for deciding the existential theory of the reals. In 29th Annual Symposium on Foundations of Computer Science, White Plains, New York, USA, 24-26 October 1988, pages 291-295, 1988.

21. J. Renegar. On the computational complexity and geometry of the first-order theory of the reals. part i: Introduction. preliminaries. the geometry of semi-algebraic sets. the decision problem for the existential theory of the reals. Journal of symbolic computation, 13(3):255-299, 1992.

22. T. Tao. Structure and randomness: pages from year one of a mathematical blog. American Mathematical Soc., 2008.

23. A. Tarski. A decision method for elementary algebra and geometry. 1951.

24. N. K. Vereshchagin. Occurrence of zero in a linear recursive sequence. Mathematical notes of the Academy of Sciences of the USSR, 38(2):609-615, 1985. 


\section{A The case of only real eigenvalues}

In this section we consider the Semialgebraic Orbit Problem in the case where the matrix $A$ has only real eigenvalues, denoted $\rho_{1}, \rho_{2}, \rho_{3}$. In this case, by converting $A$ to Jordan normal form, there exists an invertible matrix $B \in(\mathbb{A} \cap \mathbb{R})^{3 \times 3}$ such that one of the following holds:

1. $A=B^{-1}\left(\begin{array}{ccc}\rho_{1} & 0 & 0 \\ 0 & \rho_{2} & 0 \\ 0 & 0 & \rho_{3}\end{array}\right) B$, in which case $A^{n}=B^{-1}\left(\begin{array}{ccc}\rho_{1}^{n} & 0 & 0 \\ 0 & \rho_{2}^{n} & 0 \\ 0 & 0 & \rho_{3}^{n}\end{array}\right) B$.

2. $A=B^{-1}\left(\begin{array}{ccc}\rho_{1} & 1 & 0 \\ 0 & \rho_{2} & 0 \\ 0 & 0 & \rho_{3}\end{array}\right) B$ with $\rho_{1}=\rho_{2}$, in which case $A^{n}=B^{-1}\left(\begin{array}{ccc}\rho_{1}^{n} & n \rho_{1}^{n-1} & 0 \\ 0 & \rho_{1}^{n} & 0 \\ 0 & 0 & \rho_{3}^{n}\end{array}\right) B$.

3. $A=B^{-1}\left(\begin{array}{ccc}\rho_{1} & 1 & 0 \\ 0 & \rho_{2} & 1 \\ 0 & 0 & \rho_{3}\end{array}\right) B$ with $\rho_{1}=\rho_{2}=\rho_{3}$, in which case $A^{n}=B^{-1}$

$\left(\begin{array}{ccc}\rho_{1}^{n} & n \rho_{1}^{n-1} & \frac{1}{2} n(n-1) \rho_{1}^{n-2} \\ 0 & \rho_{1}^{n} & n \rho_{1}^{n-1} \\ 0 & 0 & \rho_{1}^{n}\end{array}\right) B$

In any of the forms above, we can write

$$
A^{n} s=\left(\begin{array}{l}
A_{1}(n) \rho_{1}^{n}+B_{1}(n) \rho_{2}^{n}+C_{1}(n) \rho_{3}^{n} \\
A_{2}(n) \rho_{1}^{n}+B_{2}(n) \rho_{2}^{n}+C_{2}(n) \rho_{3}^{n} \\
A_{3}(n) \rho_{1}^{n}+B_{3}(n) \rho_{2}^{n}+C_{3}(n) \rho_{3}^{n}
\end{array}\right)
$$

where the $A_{i}, B_{i}$, and $C_{i}$ are polynomials whose degree is less than the multiplicity of their corresponding eigenvalue.

In Sections 4 and 5, we reduce the problem to finding a solution to an almost self-conjugate system. In the case of real eigenvalues, the notion of almost self-conjugate is meaningless, as there are no complex numbers involved. Thus, following the analysis thereof, and plugging the entries of $A^{n} s$, we reduce the problem to solving a system of expressions of the form $\bigwedge_{J} R_{J}\left(A^{n} s\right) \sim_{J} 0$, where

$$
R_{J}\left(A^{n} s\right)=\sum_{0 \leq p_{1}, p_{2}, p_{3} \leq k} \alpha_{p_{1}, p_{2}, p_{3}}^{J}(n) \rho_{1}^{p_{1} n} \rho_{2}^{p_{2} n} \rho_{3}^{p_{3} n}
$$

for some $k \in \mathbb{N}$, and $\alpha_{p_{1}, p_{2}, p_{3}}^{J}(n)$ are polynomials.

Assuming $\rho_{1}, \rho_{2}, \rho_{3}>0$ (otherwise we can split according to odd and even $n$ ), for each such expression we can compute a bound $N \in \mathbb{N}$ based on the rate of growth of the summands, such that either for every $n>N$ the equation holds, or for every $n>N$ it does not hold.

\section{B The case where $\gamma$ is a root of unity}

We assume that $\gamma=\frac{\lambda}{|\lambda|}$ is a root of unity. That is, there exists $d \in \mathbb{N}$ such that $\gamma^{d}=1$, so we have that $\left\{\gamma^{n}: n \in \mathbb{N}\right\}=\left\{\gamma^{0}, \ldots, \gamma^{d-1}\right\}$.

Let $n \in \mathbb{N}$ and write $m=(n \bmod d)$. We can now write

$$
A^{n} s=\left(\begin{array}{l}
a_{1}|\lambda|^{n} \gamma^{m}+\overline{a_{1}}|\lambda|^{n} \bar{\gamma}^{m}+b_{1} \rho^{n} \\
a_{2}|\lambda|^{n} \gamma^{m}+\overline{a_{2}}|\lambda|^{n} \bar{\gamma}^{m}+b_{2} \rho^{n} \\
a_{3}|\lambda|^{n} \gamma^{m}+\overline{a_{3}}|\lambda|^{n} \bar{\gamma}^{m}+b_{3} \rho^{n}
\end{array}\right)=\left(\begin{array}{l}
2 \operatorname{Re}\left(a_{1} \gamma^{m}\right)|\lambda|^{n}+b_{1} \rho^{n} \\
2 \operatorname{Re}\left(a_{2} \gamma^{m}\right)|\lambda|^{n}+b_{2} \rho^{n} \\
2 \operatorname{Re}\left(a_{3} \gamma^{m}\right)|\lambda|^{n}+b_{3} \rho^{n}
\end{array}\right)
$$

Observe that there exists $n \in \mathbb{N}$ such that $A^{n} s \in T$ iff there exist $0 \leq m \leq d-1$ and $r \in \mathbb{N} \cup\{0\}$ such that $A^{r d+m} s \in T$. We can thus split our analysis according to $m \in$ $\{0, \ldots, d-1\}$. For every such $m$, we need to decide whether there exists $r \in \mathbb{N} \cup\{0\}$ such that $\left(2 \operatorname{Re}\left(a_{1} \gamma^{m}\right)|\lambda|^{m}\left(|\lambda|^{d}\right)^{r}+b_{1} \rho^{m}\left(\rho^{d}\right)^{r}\right)$

$2 \operatorname{Re}\left(a_{2} \gamma^{m}\right)|\lambda|^{m}\left(|\lambda|^{d}\right)^{r}+b_{2} \rho^{m}\left(\rho^{d}\right)^{r} \quad$ Note that $\gamma^{m},|\lambda|^{m}$ and $\rho^{m}$ are constants. Therefore, $\left.2 \operatorname{Re}\left(a_{3} \gamma^{m}\right)|\lambda|^{m}\left(|\lambda|^{d}\right)^{r}+b_{3} \rho^{m}\left(\rho^{d}\right)^{r}\right)$

these expressions contain only realalgebraic constants, the system can be viewed as a case handled in the setting of all real eigenvalues. We can thus proceed with the analysis in Section A. 
Finally, we remark that $d \leq \operatorname{deg}(\gamma)^{2}$. The proof appears in [13], and we bring it here for completeness. Since $\gamma$ is a primitive root of unity of order $d$, then the defining polynomial $p_{\gamma}$ of $\gamma$ is the $d$-th Cyclotomic polynomial, so $\operatorname{deg}(\gamma)=\Phi(d)$, where $\Phi$ is Euler's totient function. Since $\Phi(d) \geq \sqrt{d}$, we get that $d \leq \operatorname{deg}(\gamma)^{2}$. Therefore, the number of cases we consider is polynomial in the original input, and does not involve a blowup in the complexity.

\section{Change of Basis Matrices in the $3 \times 3$ case}

In this section we consider a diagonalisable matrix $A \in \mathbb{Q}^{3 \times 3}$ with complex eigenvalues. Thus, we can write $A=P D P^{-1}$ with $D=\operatorname{diag}(\lambda, \bar{\lambda}, \rho)$ with $\lambda \in \mathbb{A}$ and $\rho \in \mathbb{A} \cap \mathbb{R}$.

Note that the columns of the matrix $P$ are eigenvectors of $A$, and moreover, conjugate eigenvalues have conjugate eigenvectors and real eigenvalues have real eigenvectors. We can therefore assume

$$
P=\left(\begin{array}{lll}
a & \bar{a} & d \\
b & \bar{b} & e \\
c & \bar{c} & f
\end{array}\right)
$$

for $a, b, c \in \mathbb{A}$ and $d, e, f \in \mathbb{R} \cap \mathbb{A}$.

Lemma 4 Let $E=\operatorname{diag}\left(\delta_{1}, \delta_{2}, \delta_{3}\right)$ be a diagonal matrix, then every coordinate of $P E P^{-1}$ is of the form $\alpha \delta_{1}+\bar{\alpha} \delta_{2}+\beta \delta_{3}$, where $\alpha \in \mathbb{A}$ and $\beta \in \mathbb{A} \cap \mathbb{R}$.

Proof The proof is straightforward: we compute the matrix $P^{-1}$, and then the product $P E P^{-1}$. We leave it to the reader to verify the following: first, the determinant of $P$ is pure-imaginary, i.e., $\operatorname{det}(P)=m i$ for $m \in \mathbb{R} \cap \mathbb{A}$. Second, we have

$$
P^{-1}=\frac{1}{m i}\left(\begin{array}{ll}
f \bar{b}-e \bar{c} & d \bar{c}-f \bar{a} e \bar{a}-d \bar{b} \\
c e-b f & a f-c d b d-a e \\
b \bar{c}-c \bar{b} & c \bar{a}-a \bar{c} a \bar{b}-b \bar{a}
\end{array}\right)
$$

Finally, it is very easy (yet tedious) to verify that $P E P^{-1}$ satisfies the claim. We demonstrate by computing the coordinate $\left(P E P^{-1}\right)_{1,2}$.

We have that the first row of $P E$ is $\left(a \delta_{1}, \bar{a} \delta_{2}, d \delta_{3}\right)$, and hence

$$
\begin{aligned}
\left(P E P^{-1}\right)_{1,2} & =(P E)_{1,1} P_{1,2}^{-1}+(P E)_{1,2} P_{2,2}^{-1}+(P E)_{1,3} P_{3,2}^{-1} \\
& =\frac{1}{m i}\left(a \delta_{1}(d \bar{c}-f \bar{a})+\bar{a} \delta_{2}(a f-c d)+d \delta_{3}(c \bar{a}-a \bar{c})\right) \\
& =\frac{1}{m}\left(-i \delta_{1}(a d \bar{c}-a f \bar{a})+i \delta_{2}(\bar{a} c d-a f \bar{a})-i \delta_{3}(d c \bar{a}-d a \bar{c})\right)
\end{aligned}
$$

It is now easy to see that the coefficients of $\delta_{1}$ and $\delta_{2}$ are conjugates, and the coefficient of $\delta_{3}$ is real, as desired.

\section{Bounds on the Description Size of Points in $Z_{f}$}

We complete the analysis of Remark 2 .

Recall that $f(z)=\sum_{m=0}^{k} \beta_{m} z^{m}+\overline{\beta_{m}} \bar{z}^{m}$, and $Z_{f}=\{z: f(z)=0 \wedge|z|=1\}$. Further recall that for every $0 \leq m \leq k, \beta_{m}$ is a polynomial in $a_{1}, a_{2}, a_{3}, \overline{a_{1}}, \overline{a_{2}}, \overline{a_{3}}, b_{1}, b_{2}, b_{3}$, where all the latter are linear combinations of roots of the characteristic polynomial of $A$, and are therefore algebraic numbers of degree at most 3 and description polynomial in $\|A\|+\|s\|$.

We can now express the condition $f(z)=0$ using a quantified formula in the first-order theory of the reals by replacing each of the constants above (i.e. $a_{1}$, etc.) by their corresponding description, as per Section 2.2. It follows that in this description, there are at most 9 variables. We now employ the following result due to Renegar [21]. 
Theorem 5 (Renegar) Let $M \in \mathbb{N}$ be fixed. Let $\tau(\mathbf{y})$ be a formula of the first-order theory of the reals. Assume that the number of (free and bound) variables in $\tau(\mathbf{y})$ is bounded by $M$. Denote the degree of $\tau(\mathbf{y})$ by $d$ and the number of atomic predicates in $\tau(\mathbf{y})$ by $n$.

There is a polynomial time (polynomial in $\|\tau(\mathbf{y})\|$ ) procedure which computes an equivalent quantifier-free formula

$$
\chi(\mathbf{y})=\bigvee_{i=1}^{I} \bigwedge_{j=1}^{J_{i}} h_{i, j}(y) \sim_{i, j} 0
$$

where each $\sim_{i, j}$ is either $>$ or $=$, with the following properties:

1. Each of $I$ and $J_{i}($ for $1 \leq i \leq I)$ is bounded by $(n+d)^{O(1)}$.

2. The degree of $\chi(\mathbf{y})$ is bounded by $(n+d)^{O(1)}$.

3. The height of $\chi(\mathbf{y})$ is bounded by $2^{\|\tau(\mathbf{y})\|(n+d)}{ }^{O(1)}$.

We apply this theorem to the description of $Z_{f}$ given above, where we identify $\mathbb{C}$ with $\mathbb{R}^{2}$ so that $f$ is indeed a polynomial. Then, we obtain in polynomial time a description of $Z_{f}$. Moreover, the degrees of the entries is bounded by $\|f\|^{\mathcal{O}(1)}$ and their height is bounded by $2^{\|f\|^{\mathcal{O}(1)}}$. 